\title{
Mesonic fluctuations in a nonlocal Nambu-Jona-Lasinio model
}

\author{
Robert S. Plant* and Michael C. Birse \\ Theoretical Physics Group, Department of Physics and Astronomy, \\ University of Manchester, Manchester, M13 9PL, U.K.
}

\begin{abstract}
The effects of meson fluctuations are studied in a nonlocal generalization of the Nambu-JonaLasinio model, by including terms of next-to-leading order (NLO) in $1 / N_{c}$. In the model with only scalar and pseudoscalar interactions NLO contributions to the quark condensate are found to be very small. This is a result of cancellation between virtual mesons and Fock terms, which occurs for the parameter sets of most interest. In the quark self-energy, similar cancellations arise in the tadpole diagrams, although not in other NLO pieces which contribute at the $\sim 25 \%$ level. The effects on pion properties are also found to be small. NLO contributions from real $\pi \pi$ intermediate states increase the sigma meson mass by $\sim 30 \%$. In an extended model with vector and axial interactions, there are indications that NLO effects could be larger.
\end{abstract}

\section{INTRODUCTION}

Until recently most calculations performed with the Nambu-Jona-Lasinio (NJL) model [1] 2] have been restricted to tree-level in the mesons or, in other words, to leading order (LO) in the $1 / N_{c}$ expansion. Although the possible importance of quantum fluctuations of the mesons has been generally recognized (see, for example, Refs. [3] [5]), a satisfactory treatment of such effects has required the development of schemes that are faithful to the chiral invariance of the model. This is crucial, given that the most important motivation for studying the model is its description of the dynamical symmetry breakdown. Schemes have been introduced on the basis of an effective action method [6], an appropriate selection of Feynman diagrams [7,8] and a bosonized approximation [9].

Calculations at next-to-leading order (NLO) in $1 / N_{c}$ are certainly more involved than at LO, both analytically and numerically. However, since $1 / N_{c}$ is such a modest expansion parameter, it has rightly been seen as important to estimate the size of some NLO effects. Even if the calculations were of interest for no other reason, a check on this perturbative approach would be very valuable. Encouragingly, there seems to be a general consensus emerging from recent studies that NLO corrections are indeed relatively small for standard choices of the model parameters [6,8,10 15], at least for properties of the ground state and of the pion. A contrary view, however, has been expressed by Kleinert and Van den Bossche 16$]$.

The most compelling reason for a NLO analysis, however, is that the LO treatment of an NJL-type model neglects physical processes that are expected to be qualitatively important. For instance, several of the particles described by such models $\left(\sigma, \rho, a_{1}\right)$ are broad resonances, yet the model meson propagators at LO are purely real. At NLO the particle widths are incorporated in a natural way, by including diagrams with two-meson intermediate states in the Bethe-Salpeter equation (BSE). Although these widths can be estimated from the LO decay amplitudes, the effects on the position of a resonance require a NLO treatment. Such effects may well prove important in model descriptions of, for example, the $\rho$ meson [4, 13].

A difficulty with the model at NLO concerns the specification of model parameters associated with regularization of the loop integrals. At LO the model contains three parameters: the current quark mass, a coupling constant and a cutoff parameter that regularizes quark loops. Conventionally two of these parameters are fitted to the pion mass and decay constant, while the third is at least constrained by the value of the quark condensate. However, the regularization of the model must be further specified at NLO since meson and quark loop integrals can be regulated quite independently without affecting chiral symmetry relations [6, 8, 9, 12 14, 17]. The parameter space can be limited to some extent because the NLO corrections produce instabilities for large values of the meson cutoff [13, 17. Nonetheless the freedom to introduce another cutoff parameter produces significant uncertainty. Oertel et al. [13] have determined the meson cutoff by studying the pion electromagnetic form factor and find values well away from the region of instabilities. This is a promising result, but to put it on a firmer basis one would have to relax assumptions

${ }^{*}$ Current address: Department of Meteorology, University of Reading, Reading, RG6 6BB, U.K. 
that were made in Ref. [13] for numerical reasons]. An attractive alternative is to perform the regularization at the level of the model action rather than in the loop integrals. In this approach the regularization is specified from the outset and does not have to be imposed at each order of the expansion, a point which has been stressed by Ripka [14].

In this paper, we make a NLO analysis of a model that was originally proposed in Ref. [18]. It has subsequently been studied at LO in the meson [19,20], baryon (soliton) [21] and quark matter [11] sectors. The two-body interaction vertex is nonlocal and separable, a Gaussian form factor being associated with each quark field. Such a model successfully eliminates several of the traditional problems of the NJL model whilst nevertheless retaining much of the simplicity that is its chief merit. The separable nature of the interaction is motivated in part by instanton liquid studies [22,23]. The interaction form factors ensure the convergence of all loop integrals and so the NLO corrections are unambiguous. In addition, a practical advantage is conferred by the shape of the form factor, which allows complicated NLO diagrams to be evaluated efficiently with Gaussian numerical techniques. Such properties make this model a particularly convenient one with which to examine NLO effects. NLO quantum fluctuations of the quark condensate in a variant of this model were studied in Ref. [14]. Here we investigate the NLO treatment of mesons.

In our approach we keep the full momentum dependence of all quark loop diagrams, and avoid expanding them in powers of meson momenta. Such an expansion may be useful in the determination of pion properties, but it is not valid for heavy mesons, such as the scalar, isoscalar sigma.

\section{THE NONLOCAL MODEL}

In this section we define the model used and recall some important aspects of its behaviour at LO. This is entirely standard [2,19] and is included largely in order to establish our notation. The action of a quark model with a four-point interaction may be written

$$
\begin{gathered}
S=\int d^{4} x \bar{\psi}(x)\left(i \not \partial-m_{c}\right) \psi(x)+\sum_{i} \int \prod_{n} d^{4} x_{n} H_{i}\left(x_{1}, x_{2}, x_{3}, x_{4}\right) \\
\times \bar{\psi}\left(x_{1}\right) \Gamma_{i}^{\alpha} \psi\left(x_{3}\right) \bar{\psi}\left(x_{2}\right) \Gamma_{i \alpha} \psi\left(x_{4}\right)
\end{gathered}
$$

where $\Gamma_{i}^{\alpha}$ denotes Dirac, colour and isospin matrices. Chiral symmetry constrains certain of the possible Dirac and isospin structures to appear in particular combinations. The admissible interaction terms are

$$
\begin{aligned}
H_{1}\left(1 \otimes 1+i \gamma_{5} \tau^{a} \otimes i \gamma_{5} \tau^{a}\right), & H_{2}\left(\gamma^{\mu} \tau^{a} \otimes \gamma_{\mu} \tau^{a}+\gamma^{\mu} \gamma_{5} \tau^{a} \otimes \gamma_{\mu} \gamma_{5} \tau^{a}\right), \\
H_{3}\left(\gamma^{\mu} \otimes \gamma_{\mu}\right), & H_{4}\left(\gamma^{\mu} \gamma_{5} \otimes \gamma_{\mu} \gamma_{5}\right), \\
H_{5}\left(\tau^{a} \otimes \tau^{a}+i \gamma_{5} \otimes i \gamma_{5}\right), & H_{6}\left(\sigma_{\mu \nu} \otimes \sigma^{\mu \nu}-\sigma_{\mu \nu} \tau^{a} \otimes \sigma^{\mu \nu} \tau^{a}\right) .
\end{aligned}
$$

We shall be primarily concerned with the simplest version of the model, including the pions, and their chiral partner, through the $H_{1}$ interaction. In the usual NJL model the $H_{i}$ are simply constants. However, the use of a nonlocal interaction is appealing [18,19, 21], not least because it enables one to avoid some of the ambiguities associated with regularization. Moreover, a nonlocal interaction of separable form is suggested by studies of the instanton-liquid picture of the QCD vacuum [22,23. In this case, the momentum space form of the $H_{i}$ is

$$
H_{i}\left(p_{1}, p_{2}, p_{3}, p_{4}\right)=\frac{1}{2}(2 \pi)^{4} G_{i} f\left(p_{1}\right) f\left(p_{2}\right) f\left(p_{3}\right) f\left(p_{4}\right) \delta\left(p_{1}+p_{2}-p_{3}-p_{4}\right),
$$

with the $G_{i}$ being constants which, for the purposes of counting, we take to be $\mathcal{O}\left(1 / N_{c}\right)$. Although the instanton-liquid model [22,23] predicts a particular form for the form-factor function $f(p)$, other authors [18, 19, 21] have considered a function which is a Gaussian in Euclidean space,

$$
f\left(p_{E}\right)=\exp \left(-p_{E}^{2} / \Lambda^{2}\right)
$$

The Gaussian shape produces a good phenomenology of mesons and nucleons in the LO approximation [19,21] and is used for the numerical results in this paper.

\footnotetext{
${ }^{1}$ The $\rho$ meson was included in the model but vector and axial-vector degrees of freedom were not included as intermediate states in the NLO diagrams. In addition, $\pi a_{1}$ mixing was neglected, even though this is a LO effect.
} 
At leading order in the $1 / N_{c}$ expansion the quark propagator is dressed by a single quark-loop self-energy diagram. This results in a momentum-dependent quark "mass", $m(p)$ :

$$
\begin{gathered}
S^{-1}(p)=\not p-m(p) \\
m(p)=m_{c}+\left(m(0)-m_{c}\right) f^{2}(p),
\end{gathered}
$$

where the constant $m(0)$ is obtained from the Schwinger-Dyson equation (SDE) [18]. If $m(0)$ is sufficiently large, the poles in the quark propagator are shifted into the complex $p^{2}$ plane $14,18,19$. A feature of models with nonlocal interactions is the appearance of additional, unphysical poles in the quark propagator. For the parameter sets of most interest these are located well away from the real axis [19], but instabilities of the model ground state can appear for small values of the cutoff [14.

Mesonic bound states can be found from the poles in the $\bar{q} q$ scattering matrix, $T$. For a separable interaction, it is convenient to factor out interaction form factors of the external quark momenta and to define

$$
T\left(p_{1}, p_{2}, p_{3}, p_{4}\right)=\delta\left(p_{1}+p_{2}-p_{3}-p_{4}\right) \prod_{n} f\left(p_{n}\right) \sum_{i, j} \Gamma_{i} \hat{T}_{i j}(q) \bar{\Gamma}_{j},
$$

where $q=p_{1}-p_{3}=p_{4}-p_{2}$ denotes the total momentum of the $\bar{q} q$ pair. The notation $\bar{\Gamma}_{j}$ is needed in the longitudinal components of the vector and axial channels where, for example, $\Gamma_{V}=-\bar{\Gamma}_{V}=i \not d$. In all other cases $\Gamma_{j}$ and $\bar{\Gamma}_{j}$ are the same (see Eq. (23) of Ref. [19]). The Bethe-Salpeter equation (BSE) at LO is given by [18,19]

$$
\hat{T}(q)=G+G J(q) \hat{T}(q)
$$

where $G$ is simply a matrix of the coupling constants from the action and $J(q)$ is the matrix of polarization loop integrals

$$
J_{i j}(q)=i \operatorname{Tr} \int \frac{d^{4} p}{(2 \pi)^{4}} f^{2}\left(p_{+}\right) f^{2}\left(p_{-}\right) \Gamma_{j} S\left(p_{-}\right) \bar{\Gamma}_{i} S\left(p_{+}\right),
$$

where we have introduced $p_{ \pm}=p \pm \frac{1}{2} q$. The symbol ' $T r$ ' is used to denote a trace over flavour, colour and Dirac indices.

Close to the pole corresponding to a particular particle, the amplitude in the relevant channel may be written

$$
\frac{\bar{V}(q) \otimes V(q)}{m^{2}-q^{2}}
$$

where $V(q)$ denotes the particle vertex function. In the simplest form of the model, with only the $G_{1}$ coupling included, these functions are:

$$
V_{\pi}(q)=g_{\pi q q} i \gamma_{5} \tau^{a}, \quad V_{\sigma}(q)=g_{\sigma q q}
$$

where:

$$
\frac{1}{g_{i q q}^{2}}=\left.\frac{d J_{i i}}{d q^{2}}\right|_{q^{2}=m^{2}}
$$

The vertex functions appear in calculations of mesonic properties, such as the pion decay constant, $f_{\pi}$.

In order to obtain symmetry currents with the same divergences as the corresponding local currents in QCD, and hence to respect the corresponding Ward identities, one has to include additional nonlocal terms in the currents [18, $19,21,24]$. These arise as a consequence of the nonlocality of the action. Details of the nonlocal terms required in both vector and axial currents can be found in Refs. [18,19]. Although the method developed in those papers is not unique and there is an ambiguity in defining the transverse parts of the currents, this does not affect the longitudinal components.

As an example, consider the pion decay constant. The diagrams in Fig. 1 1 represent the contributions from the local and nonlocal pieces of the axial current. The longitudinal part of the current, $q^{\mu} A_{\mu}^{a}$, contains the following term 


$$
\begin{aligned}
\frac{i}{2(2 \pi)^{12}} G_{1} \int & \prod_{n} d^{4} p_{n} \bar{\psi}\left(p_{1}\right) i \gamma_{5} \tau^{a} \psi\left(p_{3}\right) \bar{\psi}\left(p_{2}\right) \psi\left(p_{4}\right) \delta\left(p_{1}+p_{2}+q-p_{3}-p_{4}\right) \\
& \times\left[f\left(p_{1}\right) f\left(p_{2}\right) f\left(p_{3}\right) f\left(p_{4}-q\right)+f\left(p_{1}\right) f\left(p_{2}\right) f\left(p_{3}-q\right) f\left(p_{4}\right)\right. \\
& \left.-f\left(p_{1}\right) f\left(p_{2}+q\right) f\left(p_{3}\right) f\left(p_{4}\right)-f\left(p_{1}+q\right) f\left(p_{2}\right) f\left(p_{3}\right) f\left(p_{4}\right)\right] .
\end{aligned}
$$

This term contributes to the pion decay constant since the operator $\bar{\psi}\left(p_{2}\right) \psi\left(p_{4}\right)$ has a nonzero vacuum expectation

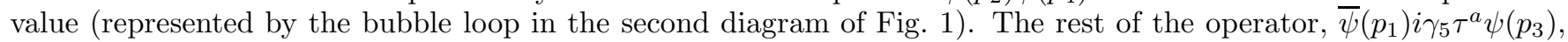
contributes to the pion-to-vacuum matrix element of the current. Such terms are needed in order to satisfy the Gell-Mann-Oakes-Renner (GMOR) relation [25], as discussed in Ref. [18], and they can make significant numerical contributions to various observables [19].

\section{NLO CONTRIBUTIONS}

\section{A. Symmetry currents}

At leading order in the $1 / N_{c}$ expansion the fields at $x_{1}$ and $x_{3}$ in the interaction term of the action (11) are always connected to the same quark loop, and similarly for the fields at $x_{2}$ and $x_{4}$. Beyond this order, there are also exchange or "Fock" terms where the fields at $x_{1}$ and $x_{4}$ are connected to the same loop. These can be constructed by first performing a Fierz transformation on the action and then using the transformed action in just the same way that one uses the original action at LO. The Fierz transformed action of this model contains the following colour-singlet terms:

$$
\begin{aligned}
& \left(4 N_{c}\right)^{-1}\left(G_{1}-2 G_{3}+2 G_{4}-G_{5}+12 G_{6}\right)\left(1 \otimes 1+i \gamma_{5} \tau^{a} \otimes i \gamma_{5} \tau^{a}\right), \\
& \left(4 N_{c}\right)^{-1}\left(-2 G_{2}+G_{3}+G_{4}\right)\left(\gamma_{\mu} \tau^{a} \otimes \gamma^{\mu} \tau^{a}+\gamma_{\mu} \gamma_{5} \tau^{a} \otimes \gamma^{\mu} \gamma_{5} \tau^{a}\right), \\
& \left(4 N_{c}\right)^{-1}\left(-2 G_{1}+6 G_{2}+G_{3}+G_{4}-2 G_{5}\right)\left(\gamma_{\mu} \otimes \gamma^{\mu}\right), \\
& \left(4 N_{c}\right)^{-1}\left(2 G_{1}+6 G_{2}+G_{3}+G_{4}+2 G_{5}\right)\left(\gamma_{\mu} \gamma_{5} \otimes \gamma^{\mu} \gamma_{5}\right), \\
& \left(4 N_{c}\right)^{-1}\left(-G_{1}-2 G_{3}+2 G_{4}+G_{5}-12 G_{6}\right)\left(\tau^{a} \otimes \tau^{a}+i \gamma_{5} \otimes i \gamma_{5}\right), \\
& \left(8 N_{c}\right)^{-1}\left(G_{1}-G_{5}-4 G_{6}\right)\left(\sigma_{\mu \nu} \otimes \sigma^{\mu \nu}-\sigma_{\mu \nu} \tau^{a} \otimes \sigma^{\mu \nu} \tau^{a}\right) .
\end{aligned}
$$

(There are also colour-octet terms which we do not use here.)

The nonlocal terms in the symmetry currents of the model also involve four quark fields and so will also be subject to exchange effects at NLO. These Fock pieces in the currents will introduce further ambiguity through the definition of their transverse parts. One way to construct the NLO current terms is to take the Fierz transformed action and to apply the same method as was used in Refs. [18, 19] to form the nonlocal currents from the original model action. This method leads to nonlocal NLO current terms of the same structures as those presented in Ref. [19], with the appropriate coupling constants in the original terms replaced by the corresponding combinations of couplings from the Fierz transformed action. For example, as well as a term $G_{1}\left(i \gamma_{5} \tau^{a} \otimes 1\right)$ in the nonlocal axial current constructed from the original action (see Eq. (13)), there is a Fock term of the same structure where $G_{1}$ is replaced by $\left(4 N_{c}\right)^{-1}\left(G_{1}-2 G_{3}+2 G_{4}-G_{5}+12 G_{6}\right)$.

An alternative approach to constructing the Fock terms of the model's currents is simply to Fierz transform the LO currents of Ref. [19]. The differences between this procedure and the one outlined above (where the Noetherlike method for deriving a symmetry current is applied after the Fierz transformation) lie in the combinations of form factors which are present in the currents. However, these affect only the transverse parts of the currents; both definitions satisfy the appropriate Ward identities. The equivalence of the longitudinal components has been checked explicitly [26]. In practice, the currents obtained from the Fierz-transformed action are somewhat easier to work with, since they retain the same structures as the nonlocal terms in the LO currents, with appropriate substitutions for the overall coefficients.

\section{B. Quark propagator}

We are now in a position to consider the quark and meson propagators at NLO. Like Oertel et al. 12, 13, 15, and Ripka 14] we use a strict NLO scheme in which all propagators and matrix elements are expanded to NLO in $1 / N_{c}$. This scheme has also been presented by Dmitrašinović et al. [8], who themselves preferred to adopt an alternative

scheme where certain NLO terms in the quark self-energy are treated self-consistently in the quark Schwinger-Dyson equation. Their scheme resums a subset of terms to all orders in $1 / N_{c}$. Both schemes satisfy chiral low-energy 
theorems [8,13]. The Feynman diagrams required by the strict NLO scheme in the nonlocal NJL model are briefly presented below. |7

At NLO the quark Schwinger-Dyson equation is coupled to the meson Bethe-Salpeter equation and takes the form

$$
\begin{aligned}
S_{F}^{-1}(p)=\not p-m_{c}- & i G_{1} f^{2}(p) \operatorname{Tr} \int \frac{d^{4} k}{(2 \pi)^{4}} S_{F}(k) f^{2}(k) \\
& +i f^{2}(p) \sum_{i, j} \int \frac{d^{4} k}{(2 \pi)^{4}} \hat{T}_{i j}(k) \Gamma_{i} S_{F}(p-k) \bar{\Gamma}_{j} f^{2}(p-k),
\end{aligned}
$$

where $\hat{T}_{i j}(k)$ denotes the element of the $\bar{q} q$ scattering matrix which describes scattering from the state with matrix structure $\Gamma_{j}$ to the state with structure $\Gamma_{i}$. This matrix is diagonal apart from the mixing between the pseudoscalar and axial channels which is present in the extended model with vector and axial interactions. It is to be understood as the LO scattering matrix, which is of order $N_{c}^{-1}$ (see Eq. (8)).

Although the SDE as written above contains all of the required terms at LO and NLO, it also includes some unwanted higher order terms. The NLO terms can be obtained by replacing the quark propagator in the final integral by its LO form, and by expanding the propagator in the quark "bubble" integral to NLO:

$$
S_{F}(p) \simeq S(p)+S(p) \Sigma^{(1)}(p) S(p),
$$

where $\Sigma^{(1)}$ denotes the NLO contribution to the quark self-energy. This leads to an equation for $\Sigma^{(1)}$ of the form

$$
\begin{aligned}
\Sigma^{(1)}(p)= & i G_{1} f^{2}(p) \operatorname{Tr} \int \frac{d^{4} k}{(2 \pi)^{4}} S(k) \Sigma^{(1)}(k) S(k) f^{2}(k) \\
& -i f^{2}(p) \sum_{i, j} \int \frac{d^{4} k}{(2 \pi)^{4}} \hat{T}_{i j}(k) \Gamma_{i} S(p-k) \bar{\Gamma}_{j} f^{2}(p-k) .
\end{aligned}
$$

The first term comes from an insertion of the NLO self-energy into the quark bubble diagram. The second, involving the scattering matrix, includes both Fock terms and dressing of the quark by virtual mesons.

The separable nature of the interaction means that this equation can be solved straightforwardly to get

$$
\Sigma^{(1)}(p)=f^{2}(p) C-i f^{2}(p) \sum_{i, j} \int \frac{d^{4} k}{(2 \pi)^{4}} \hat{T}_{i j}(k) \Gamma_{i} S(p-k) \bar{\Gamma}_{j} f^{2}(p-k),
$$

where

$$
\begin{gathered}
C=\frac{G_{1}}{1-G_{1} J_{\sigma \sigma}(0)} \sum_{i, j} \operatorname{Tr} \int \frac{d^{4} k}{(2 \pi)^{4}} \int \frac{d^{4} \ell}{(2 \pi)^{4}} \hat{T}_{i j}(k-\ell) \\
\times S(k) \Gamma_{i} S(\ell) \bar{\Gamma}_{j} S(k) f^{4}(k) f^{2}(\ell) .
\end{gathered}
$$

The two terms correspond to the tadpole and meson cloud diagrams shown in Fig. 2. The tadpole diagram is responsible for the contribution $f^{2}(p) C$. It includes the exchange of a zero-momentum sigma meson between the quark and a virtual meson. Since $C$ is a momentum-independent constant, this contribution has the same form as the LO quark self-energy. The other diagram describes the emission and subsequent reabsorption of a virtual meson. It has a nontrivial dependence on the momentum of the quark and generates a wave function renormalization as well as a scalar term. Note that although we shall often refer to the upper double line in such diagrams as a meson propagator, it is really a $\bar{q} q$ scattering amplitude and so the diagrams contain Fock terms as well as virtual meson contributions.

\section{Meson propagators}

At LO, the mesonic bound states are constructed from the ladder Bethe-Salpeter equation (Eq. (8)). The NLO extension of the BSE can be expressed in terms of corrections to the basic two-quark loop of Eq. (9). If the quantities

\footnotetext{
${ }^{2}$ We refer to a loop integral containing $n$ LO quark propagators as an $n$-quark loop.
} 
$J_{i j}$ are redefined to include these NLO contributions, the scattering matrix can still be written in the form of Eqs. (7) and (8).

An obvious set of NLO terms in a polarization loop integral $J_{i j}(q)$ consists of insertions of the NLO quark selfenergies of Fig. 2 on either the quark or antiquark line. These contribute

$$
\begin{aligned}
& i \operatorname{Tr} \int \frac{d^{4} p}{(2 \pi)^{4}} \Gamma_{j} S\left(p_{-}\right) \Sigma^{(1)}\left(p_{-}\right) S\left(p_{-}\right) \bar{\Gamma}_{i} S\left(p_{+}\right) f^{2}\left(p_{-}\right) f^{2}\left(p_{+}\right) \\
+ & i \operatorname{Tr} \int \frac{d^{4} p}{(2 \pi)^{4}} \Gamma_{j} S\left(p_{-}\right) \bar{\Gamma}_{i} S\left(p_{+}\right) \Sigma^{(1)}\left(p_{+}\right) S\left(p_{+}\right) f^{2}\left(p_{-}\right) f^{2}\left(p_{+}\right) .
\end{aligned}
$$

A second kind of NLO contribution arises from the exchange of a $t$-channel virtual meson between the quark and the antiquark. The corresponding diagram is shown in Fig. 3 and makes the following contribution to $J_{i j}(q)$ :

$$
\begin{aligned}
\sum_{l, m} \operatorname{Tr} \int \frac{d^{4} p}{(2 \pi)^{4}} \int \frac{d^{4} k}{(2 \pi)^{4}} \hat{T}_{l m}(p-k) \Gamma_{j} S\left(k_{-}\right) \Gamma_{l} S\left(p_{-}\right) \bar{\Gamma}_{i} S\left(p_{+}\right) \bar{\Gamma}_{m} S\left(k_{+}\right) \\
\times f^{2}\left(k_{-}\right) f^{2}\left(k_{+}\right) f^{2}\left(p_{-}\right) f^{2}\left(p_{+}\right) .
\end{aligned}
$$

Finally, there are NLO contributions that involve intermediate two-meson states, represented by the second diagram in Fig. 3. These allow for the instability of a meson against two-body decays and so can introduce an imaginary part in the corresponding propagator above the threshold energy for the two-particle final states. They are constructed by connecting two LO three-meson vertices with two meson propagators. The resulting imaginary part of a meson mass can be expressed in terms of the square of the LO decay amplitude. These contributions also generate a shift in the real part of the mass which involves a new type of loop integral.

In writing explicit expressions for these contributions, it is convenient to introduce functions $L_{i j, k}$ and $\bar{L}_{i, j k}$ to describe the LO three-point vertices. The function $L_{i j, k}$ describes the process $k \rightarrow i j$ and is defined as

$$
\begin{aligned}
& L_{i j, k}\left(q_{1}, q_{2}, q\right)= i \operatorname{Tr} \int \frac{d^{4} p}{(2 \pi)^{4}} \Gamma_{k} S\left(p_{-}\right) \bar{\Gamma}_{j} S\left(p-\frac{1}{2}\left(q_{1}-q_{2}\right)\right) \bar{\Gamma}_{i} S\left(p_{+}\right) \\
& \times f^{2}\left(p_{-}\right) f^{2}\left(p_{+}\right) f^{2}\left(p-\frac{1}{2}\left(q_{1}-q_{2}\right)\right) \\
&+i \operatorname{Tr} \int \frac{d^{4} p}{(2 \pi)^{4}} \Gamma_{k} S\left(p_{-}\right) \bar{\Gamma}_{i} S\left(p+\frac{1}{2}\left(q_{1}-q_{2}\right)\right) \bar{\Gamma}_{j} S\left(p_{+}\right) \\
& \times f^{2}\left(p_{-}\right) f^{2}\left(p_{+}\right) f^{2}\left(p+\frac{1}{2}\left(q_{1}-q_{2}\right)\right),
\end{aligned}
$$

while $\bar{L}_{i, j k}$ describes the process $j k \rightarrow i$,

$$
\begin{aligned}
\bar{L}_{i, j k}\left(q, q_{1}, q_{2}\right)=i \operatorname{Tr} \int \frac{d^{4} p}{(2 \pi)^{4}} \bar{\Gamma}_{i} S\left(p_{+}\right) \Gamma_{j} S\left(p-\frac{1}{2}\left(q_{1}-q_{2}\right)\right) \Gamma_{k} S\left(p_{-}\right) \\
\times f^{2}\left(p_{-}\right) f^{2}\left(p_{+}\right) f^{2}\left(p-\frac{1}{2}\left(q_{1}-q_{2}\right)\right) \\
+i \operatorname{Tr} \int \frac{d^{4} p}{(2 \pi)^{4}} \bar{\Gamma}_{i} S\left(p_{+}\right) \Gamma_{k} S\left(p+\frac{1}{2}\left(q_{1}-q_{2}\right)\right) \Gamma_{j} S\left(p_{-}\right) \\
\quad \times f^{2}\left(p_{-}\right) f^{2}\left(p_{+}\right) f^{2}\left(p+\frac{1}{2}\left(q_{1}-q_{2}\right)\right) .
\end{aligned}
$$

The contribution to $J_{i j}(q)$ from the two-meson diagrams can then be written in the form

$$
-\frac{i}{2} \sum_{k, l, m, n} \int \frac{d^{4} p}{(2 \pi)^{4}} \bar{L}_{i, k m}\left(q, p_{+},-p_{-}\right) \hat{T}_{k l}\left(p_{+}\right) \hat{T}_{m n}\left(-p_{-}\right) L_{l n, j}\left(p_{+},-p_{-}, q\right)
$$

Once the full NLO matrix $J^{(1)}$ has been constructed, we can use it to find the corrections to meson masses and meson-quark couplings. As an example, consider the pion in the version of the model without vector and axial couplings. The pion pole is located at

$$
1-G_{1}\left[J_{\pi \pi}^{(0)}\left(q^{2}\right)+J_{\pi \pi}^{(1)}\left(q^{2}\right)\right]=0,
$$

where superscripts (0) and (1) denote LO and NLO terms. The coupling to quarks (Eq. (12)) is given by 


$$
\left(g_{\pi q q}^{(0)}+g_{\pi q q}^{(1)}\right)^{-2}=\left.\frac{d\left(J_{\pi \pi}^{(0)}+J_{\pi \pi}^{(1)}\right)}{d q^{2}}\right|_{q^{2}=m_{\pi}^{2}} .
$$

From these we get the squared pion mass to NLO,

$$
m_{\pi}^{2}=m_{\pi}^{(0) 2}\left[1+g_{\pi q q}^{(0) 2} \frac{d J_{\pi \pi}^{(1)}}{d q^{2}}\right]_{q^{2}=m_{\pi}^{(0) 2}}-g_{\pi q q}^{(0) 2} J_{\pi \pi}^{(1)}\left(m_{\pi}^{(0) 2}\right),
$$

and the pion-quark coupling,

$$
g_{\pi q q}^{(1)}=-\frac{g_{\pi q q}^{(0) 3}}{2}\left[2 m_{\pi}^{(0)} m_{\pi}^{(1)} \frac{d^{2} J_{\pi \pi}^{(0)}}{d\left(q^{2}\right)^{2}}+\frac{d J_{\pi \pi}^{(1)}}{d q^{2}}\right]_{q^{2}=m_{\pi}^{(0) 2}}
$$

\section{Meson coupling to a current}

In this section, we consider a NLO determination of the coupling between a meson and an external current, taking the pion decay constant as an example. The situation is more complicated in a nonlocal than a local model [6,8] because there are two kinds of contribution to be considered, arising from the local and nonlocal parts of the symmetry current (see Sec. II).

The LO diagrams are shown in Fig. 1. At NLO several of the extra contributions can be identified straightforwardly, either by inserting the NLO self-energy on any one of the quark lines in these diagrams or by using the NLO contribution to the pion vertex function. Other contributions are analogous to those in the BSE at NLO. These involve either a $t$-channel meson exchange in the two-quark loop or intermediate two-meson states. They are shown in Fig. 1 .

The remaining NLO contributions arise solely from the nonlocal piece of the current. The first of these are Fock terms arising from the exchange of two of the quark lines at a nonlocal current vertex, as shown in Fig. 5 . Since they contain only a single colour trace, as indicated by separating the quark lines associated with each of the $\bar{\psi} \Gamma \psi$ factors, this diagram is suppressed by one power of $N_{c}$ compared with the corresponding LO terms. Although, as noted above, there is an ambiguity in defining the transverse components of such Fock terms, this does not affect the calculation of the pion decay constant.

Finally there are two NLO contributions which can be thought of as arising from nonlocal current couplings to virtual mesons. They are shown in Fig. 6. The first (a) is somewhat similar to diagram 1 (d), except that the coupling of the nonlocal current to the virtual mesons involves both the quark and the antiquark instead of a separate quark bubble. This is analogous to the two-body diagrams that contribute at LO to several of the electromagnetic amplitudes described in Ref. [19]. The other diagram (b) can be thought of as coupling the current at a meson-quark vertex.

Although the NLO contributions to the pion decay constant involve rather a large number of diagrams, in practice the situation can be significantly simplified as a result of cancellations amongst them [26. A complete description of these cancellations would be somewhat lengthy and so we offer a brief summary in Appendix A. We have also checked the consistency of our treatment by verifying that the GMOR relation [25] holds to NLO (see Appendix B).

\section{NUMERICAL RESULTS}

\section{A. Model parameters}

The multiple integrals involved in the NLO diagrams can be rather time-consuming to evaluate numerically to good accuracy. We have therefore chosen to fit the model parameters at LO and then to calculate the NLO changes to observables. In fact, for the parameter sets of most interest, we find that the corrections are small for the observables used to fix the parameters. Thus, our results should not be very different from those of a full NLO fit.

We fit the parameters at LO as in Ref. [19. In the simpler version of the model with no vector and axial couplings, we fit two parameters to the pion mass and decay constant. This leaves one free parameter which we characterize 
in terms of $m_{0}(0)$, the quark self-energy at zero momentum in the chiral limit. Details of the sets used are given ${ }^{3}$ in Table I. Values of some quantities calculated at LO with these parameter sets are also given in the table. They are qualitatively similar to those obtained in the extended version of the model [19]. For the parameter sets with $m_{0}(0)=300 \mathrm{MeV}$ and larger, the quark propagator has no poles on the real axis and so single quarks do not appear as physical states 18 . 19 .

Although most of our work at NLO has used this simpler model, we also show a few results for the extended model including vector and axial couplings. For these we use a parameter set with $m_{0}(0)=300 \mathrm{MeV}$, which is intermediate between the two cases studied at LO in Ref. [19].

\section{B. Quark condensate}

The NLO term in the quark condensate can be evaluated as the sum of two contributions. These correspond to the two self-energy diagrams of Fig. 2 where the external quark line is formed into a closed loop with a local scalar insertion [7]. By comparing the values for the condensate at NLO in Table II with the LO values in Table [I, we see that the NLO contributions are very small. This may seem surprising since virtual pions would be expected to make a large (positive) contribution to the condensate. However it should be recalled that the meson "propagators" in the NLO diagrams also contain the Fock terms of the two-body interaction. These make a negative contribution to the condensate which tends to cancel the contributions of virtual mesons. This agrees with the findings of Ref. [14] in a similar model. In the local version of the NJL model, the condensate is also little altered at NLO if proper time regularization of the quark loops is used [6], but in the $O(4)$ scheme there can be appreciable changes at NLO. Including only the pole pieces of the meson propagators, significant shifts in the condensate are also found with $O(3)$ regularization [27].

\section{Quark propagator}

The quark self-energy at NLO is given by Eqs. (18) and (19). Its evaluation requires integrating over the scattering matrix $\hat{T}$. Evaluating the $\bar{q} q$ loop integrals $J$ for each point in the mesonic loop integral is very time consuming. We have therefore found it convenient to parameterize the $J$ 's in terms of a set of smoothly-varying functions. Working in Euclidean space, and with the momentum routings of Eqs. (18) and (19), the J's always have a spacelike momentum argument and so are smoothly-varying functions. We have found that they are well represented by expansions in Chebyshev polynomials of the variable $x=\exp \left(q^{2} / \Lambda^{2}\right)$.

We write the inverse quark propagator as

$$
S_{F}^{-1}(p)=(1+a(p)) p-b(p) .
$$

The functions $a(p)$ and $b(p)$ are shown in Figs. 7 and 8, for both spacelike and timelike momenta. They are plotted only up to the energy given by the real part of the complex pole in the LO quark propagator. Above that energy there are pseudo-threshold effects in the model associated with the continuation from Euclidean to Minkowski space [19].

At LO $a(p)=0$ and there is no wave function renormalization. The NLO contributions to the coefficient $\not p$ range up to about 0.25 , which is consistent with the expected magnitude of $1 / N_{c}$ corrections. An interesting aspect of the results for $a(p)$ is the appearance of a sudden dip just before the pseudo-threshold energy. It would be interesting to examine the behaviour of the function above this energy, although that would require a detailed analysis of the quark pole structure at NLO, which is beyond the scope of the present work. Also plotted are the individual contributions to $a(p)$ which arise from dressing the quark line with virtual pions and with virtual sigma mesons. The pion cloud is obviously the dominant contributor. Since its propagator has a pole at small timelike momentum, one expects the $T$ matrix in the pseudoscalar channel to be large at modest values of spacelike momenta (the region which dominates the NLO integrals). Note also that the pion contributions contain an extra factor of three due to isospin.

The coefficient of the unit matrix in the quark self-energy at NLO receives contributions from both the tadpole and the meson-cloud diagrams of Fig. 2. The results in Fig. 8 show that the tadpole contribution is in fact rather small,

\footnotetext{
${ }^{3}$ Note that the parameters given differ slightly from those quoted in Ref. 18 where a very similar fit was made at the same values of $m_{0}(0)$. This is simply because the calculations of Ref. [18] were performed within the chiral expansion of the model.
} 
$C \ll m(0)$. The meson-cloud contributions are rather more significant, increasing $b(0)$ by about $25 \%$ (again consistent with expectations for a $1 / N_{c}$ effect). The net NLO shift in the quark "mass" at zero momentum $b(0) /(1+a(0))$ is therefore fairly modest, $\sim 15 \%$. Quark dressing by meson clouds in the local NJL model has been investigated in Ref. [3]. It was found that the pion cloud tends to increase $b(p)$ but that this is partially cancelled by the sigma cloud. The nonlocal model studied here supports that conclusion and is able to place it on a firmer footing since there are no ambiguities associated with the meson loop regularization't?

Although the tadpole diagrams in the NLO quark self-energy are not numerically significant, $C$ controls the NLO changes to the pion mass and decay constant. This can be seen in the chiral expansions of these quantities described in Appendix B. One might therefore wonder whether there is some physical reason why its chiral limit $C_{0}$ turns out to be small. For the parameter set with $m_{0}(0)=200 \mathrm{MeV}$ Table II shows that the pion tadpole makes a contribution of about $45 \%$ of the LO chiral quark mass, but that this is cancelled to a large extent by the sigma tadpole. Such cancellations do not persist at larger $m_{0}(0)$, where the pion tadpole changes sign. However for these parameter sets both tadpole contributions are small. In these cases it is a cancellation between "true" virtual meson effects and Fock terms (similar to what happens in the quark condensate) which is responsible for the small net effect.

In the alternative scheme of Ref. 8], the $1 / N_{c}$ contribution of the tadpole diagram in Fig. 2 is resummed to all orders when the SDE is solved self-consistently. In contrast, the momentum-dependent contribution of the mesoncloud diagram is treated perturbatively. This partial resummation is motivated by an expectation that contributions from the tadpole diagrams are larger than the other NLO effects 8 . However we find that such an expectation is not fulfilled in the nonlocal model; in fact we find that the contribution of the tadpole diagram is small compared to that of the meson-cloud diagram. We thus see no compelling reason to resum this contribution, and so we can avoid possible problems with the self-consistent scheme which have been noted in the context of the local NJL model [15].

In the extended version of the model there are additional contributions to the NLO quark self-energy from tadpole and meson-cloud diagrams with other mesons. Although a calculation of the properties of these mesons at NLO is beyond the scope of the present work, it is straightforward to evaluate their contributions to the quark self-energy. As in the simpler model, we find that there is only a modest NLO change to the condensate and that the constant $C$ is small. Pseudoscalar-axial mixing makes a significant difference to the contribution from the pion cloud to the function $b(p)$, but this is largely cancelled by the vector mesons to leave a function very similar to that found in the simpler version of the model.

The function $a(p)$ in the extended model is shown in Fig. 9. Mixing in the pion channel and the clouds of the spin-1 states are again important. In $a(p)$ these effects reinforce each other and so the function is significantly larger than in the simpler model. An interesting difference between Figs. 7 and 9 is the absence in the latter case of a dip just before the LO pseudo-threshold energy. This is eliminated in the overall result due to the contributions from the longitudinal components of the vector and axial propagators. In particular, the steep drop in the pion contribution which occurs in the simpler model is removed by mixing with the axial channel. We note that the local NJL model has been used in Ref. [13] to describe the $\rho$ meson at NLO, assuming that the effects of $\pi a_{1}$ mixing and intermediate spin-1 states could be neglected. The results presented here raise some doubts about those assumptions. Of course definitive statements about the quark propagator in the present model cannot be made without a full NLO analysis of meson properties in order to refit the model parameters. Since $1 / N_{c}$ effects could alter meson masses significantly, the parameters used here may not constitute a reasonable choice for the extended model at NLO.

\section{Pion properties}

The denominator of the $\bar{q} q$ scattering amplitude in the pion channel, Eq. (25), is shown in Fig. 10. For low energies, less than about $250 \mathrm{MeV}$, the differences between the LO and NLO curves are very small. This implies that NLO contributions to both the pion mass and the pion-quark coupling are extremely small.

Although NLO contributions to the pion mass must cancel in the chiral limit, as described in Appendix B, such cancellations do not influence higher order terms in the chiral expansion. The smallness of the NLO terms in the pion propagator is another consequence of the cancellation between virtual mesons and Fock terms, as we saw above in the case of the quark propagator. In particular, all of the contributions to the pion mass and decay constant that are proportional to the NLO piece of the quark condensate or to $C$ are small. (More details of these quantities are given in the appendices.) This supports the usual LO treatment of the pion in this type of model and it also justifies our

\footnotetext{
${ }^{4}$ Note that the tadpole contributions were not included in Ref. [3]. Moreover the model meson propagators were approximated by the canonical forms for point-like bosons.
} 
use of model parameters fitted to the LO pion properties in these NLO computations. Actual determinations of the NLO shifts in $m_{\pi}$ and $f_{\pi}$ have not been made in this work since they are sufficiently small (not more than a couple of $\mathrm{MeV}$ ) that our numerical integration procedures would have to be refined in order to yield accurate values.

At higher energies, beyond those shown in Fig. 10, NLO contributions in the pion channel do start to become significant. When the $\pi \sigma$ channel opens, the denominator develops an imaginary part. Shortly before the pseudothreshold energy (twice the real part of the complex energy of the pole in the quark propagator [19]) is reached, the NLO terms become sufficiently important to change the qualitative behaviour of the pion Bethe-Salpeter amplitude. The slope of the denominator changes sign and there is even a second zero of Eq. (25). This is a potentially worrying feature since it corresponds to an unphysical pole in the amplitude, whose residue has the wrong sign. A similar pole has been found in the local NJL model [12]. In both models the undesirable high-energy behaviour is caused by the insertions of NLO quark self-energies on the quark lines of the basic polarization loop integral. However it is perhaps worth noting that this behaviour is reminiscent of that exhibited by $a(p)$ at high energies in Fig. 7 . As mentioned in Sec. IV , this behaviour is not present in the model with vector and axial mesons and it is thus possible that the unphysical pion pole may be removed by extending the model to include interactions in these channels.

\section{E. Sigma properties}

The interpretation of the scalar, isoscalar sigma meson in dynamical quark models is complicated by the fact that it does not correspond to an experimentally well-determined resonance. The problem, not just for models of such a resonance but also for phenomenological determinations of its properties, is its very strong coupling to the twopion channel. Indeed, while some phenomenological analyses favour a scalar, isoscalar resonance at around $1 \mathrm{GeV}$ [29], more recently others have found resonances at $600 \mathrm{MeV}$ or lower [30] (for reviews and more complete lists of references see Refs. 31 33]). The strong coupling to pions means that any resonance must form a very broad structure. Consequently the corresponding pole in the scattering amplitude must lie far from the real axis, which makes its exact position hard to determine in a model-independent way [32].

This strong coupling to two pions also means that a bare $\bar{q} q$ state in the scalar, isoscalar channel should not be directly compared with any phenomenological resonance. The effects of the two-pion channel need to be included. In the present model, the leading effects of this type arise from the second diagram of Fig. 3.

At LO in $1 / N_{c}$, the nonlocal NJL model leads to a $\bar{q} q$ sigma meson with a mass of less than $500 \mathrm{MeV}$. This is similar to the local model, where the sigma lies on the $\bar{q} q$ threshold [1].2]. Some of the properties of the state are listed in Table III. It is strongly coupled to two pions, although its low mass means that its decay width is less than about $160 \mathrm{MeV}$. Although wide, this is still narrower than phenomenological fits which tend to give widths of at least 300 $\mathrm{MeV}$, even for low sigma masses 30 33.

The real part of the denominator of the scattering amplitude at NLO is shown in Fig. 11. Above the two-pion threshold, there is an imaginary part generated by the contribution to $J_{\sigma \sigma}$ from the second diagram of Fig. 3 . This can be estimated from the LO decay amplitude using the Cutkosky cutting rules [28] and it is shown in Fig. 12 along with the results from a direct numerical evaluation. Some care must be taken in the evaluation of this diagram owing to singularities of the scattering matrices in the integrand above the decay threshold. We have regulated Eq. 24) by replacing $\hat{T}_{\pi \pi}$ with $G_{1}\left(1-G_{1} J_{\pi \pi}^{(0)}-i \epsilon\right)^{-1}$ and linearly extrapolated our results to $\epsilon=0$, based on several computations for $\epsilon \sim 10^{-3}$.

The values listed in Table III for the mass of the sigma at NLO are for the mass defined as the zero of the real part of the denominator. Although this is a commonly used choice, it might be better to use the real part of the complex energy of the pole in the scattering matrix, as discussed by Pennington [32]. The choice used here is purely one of convenience. In any case the width of the state we find is not so large that we would expect substantial differences between the two definitions.

We find that the sigma mass is increased from its LO value by about 30\%. Below the two-pion threshold the largest NLO contribution to $J_{\sigma \sigma}$ arises from virtual two-pion intermediate states. This is partially cancelled by the NLO quark self-energy insertions to leave a modest net increase in $J_{\sigma \sigma}$ for most parameter sets. On their own these effects of virtual mesons would tend to reduce the sigma mass. However, above the threshold, real two-pion states make a substantial contribution with the opposite sign, leading to the increase to the sigma mass. This is consistent with the qualitative expectation that such states should be important for modelling the sigma.

NLO corrections to the sigma mass have been calculated by Pallante [9] using a derivative expansion of the bosonized NJL model. In that framework the corrections were found to be large and negative, prompting that author to speculate that the $1 / N_{c}$ expansion might break down for the mass of this state. In the local NJL model, the large negative corrections to $1-G_{1} J_{\sigma \sigma}$ persist in calculations to all orders in momentum if one uses Pauli-Villars regularization of the quark loops and a sharp cutoff for the meson loop momenta 15,34. The shifts in this case are sufficiently large to 
move the model sigma pole to negative $q^{2}$, producing an unstable vacuum. In both of these calculations, however, the results in the scalar sector were very sensitive to the additional cut-off parameter needed to regularize meson loops in the local model. In contrast, the magnitude of the NLO mass shift which we obtain in the nonlocal model is consistent with expectations for a $1 / N_{c}$ correction. Thus it would appear that the details of the treatment of high-momentum states are important in NJL-type models.

The imaginary part of the polarization loop $J_{\sigma \sigma}$ is shown in Fig. 12 and compared with the estimate obtained by applying the Cutkosky cutting rules to graphs with two-pion intermediate states. The difference between these curves is caused by the analytic structure of the quark propagator, which contains additional, unphysical poles (Sec. [II). It is reassuring to note that these extra poles are located sufficiently far from the low-energy region of interest that they do not have a large effect on the imaginary part. For other parameter sets, with large $m_{0}(0)$, the extra poles lie closer to the physical region 19 and may have some influence on properties of the sigma meson at NLO. Since the model predictions may be unreliable in these cases, we have preferred to quote only upper bounds on the sigma mass for some parameter sets in Table III, limited by the position of the first pole in the LO quark propagator.

For energies up to $\sim 600 \mathrm{MeV}$, the three-quark integral in the $\sigma \pi \pi$ vertex function is only weakly dependent on the energy. Therefore the variation of $\operatorname{Im}\left(\mathrm{J}_{\sigma \sigma}\right)$ at such energies is primarily a consequence the available two-pion phase space. At higher energies the coupling to two pions becomes much weaker 5 . This is similar to the behaviour of the coupling of the sigma to two pions observed in the quark model studied in Ref. [35] (where the scalar mass was taken as a free parameter and chosen with the intention of interpreting the model scalar resonance as a heavy, narrow state).

We note that a two-flavour NJL model of pions and sigma mesons is insufficient for a fully realistic description of the spectrum of scalar mesons. One must also take account of strangeness, radial excitations, mixing with $\bar{q} q$ molecules or glueballs, and decays to four pion states. A recent attempt to include such effects can be found in Ref. [36].

\section{CONCLUSIONS}

We have investigated the effects of meson fluctuations on quark and meson properties in a nonlocal NJL model [18,19 by treating it to NLO in $1 / N_{c}$. The two-body interaction between quarks has a separable form, similar to that suggested by instanton liquid studies [22,23. At LO the model has been shown to provide successful descriptions of mesons and baryons 19,21. The interaction form factors regulate both quark and meson loops in a natural way. As a result, no new parameters emerge at NLO and the contributions are unambiguous. This makes the model a particularly suitable one in which to study NLO effects.

The contributions to the Schwinger-Dyson and Bethe-Salpeter equations at NLO contain the same structures as those in the local model [6.8], apart from the presence of interaction form factors. The NLO pieces include the dressing of quarks and mesons with virtual mesons and the coupling of mesons to two-meson states. They also include the exchange or Fock terms for the basic interaction between quarks.

With a nonlocal interaction between the quarks, there are two-body terms in the symmetry currents [18, 19, 24] and these must be taken into account in the NLO contributions to the couplings of mesons to external currents. We have obtained the NLO pieces of the currents by first Fierz transforming our interaction and then applying the technique of Refs. [18, 19]. As a consistency check on our treatment we have verified that the GMOR relation holds at NLO.

In the simple version of the model with only scalar and pseudoscalar interactions we find that the NLO contributions to a number of quantities are small for the parameter sets of most interest (the ones with LO quark self-energies of about $300-500 \mathrm{MeV}$ ). These quantities include the quark condensate and the tadpole piece of the quark self-energy. The small size of the NLO pieces is a consequence of cancellations between the effects of virtual mesons and Fock terms, as also noted by Ripka [14]. Similar cancellations mean that the NLO contributions to the pion mass and decay constant are also small. At NLO these quantities depend not just on the NLO pieces of the quark condensate and pion-quark coupling, but also on the tadpole piece of the quark self-energy. The fact that this NLO tadpole energy is small is crucial to the smallness of the corrections to pion properties. In other cases, such as the "meson cloud" pieces of the quark-self energy, NLO contributions are larger. However they typically contribute at the $\sim 25 \%$ level and so give reason to hope that the $1 / N_{c}$ expansion is a useful organizing principle for this model.

In this context, it is worth making a remark about the recent claim of Kleinert and Van den Bossche [16] that meson fluctuation effects in the NJL model are large enough to restore manifest chiral symmetry, at least for small

\footnotetext{
${ }^{5}$ This supports a suggestion made in Ref. 19] that the weak coupling of the scalar channel to two pions above the sigma mass means that the broad width found for $a_{1} \rightarrow \sigma \pi$ in the model need not be inconsistent with the experimental observation of a small width for $a_{1} \rightarrow \pi(\pi \pi)_{s}$.
} 
values of $N_{c}$. Although our approach is based on a loop expansion, one might have expected to see large decreases in the magnitudes of quantities like the quark condensate, the quark self-energy and the pion decay constant if mesonic fluctuations were driving the system towards symmetry restoration. In fact pionic fluctuations do make significant contributions in this direction, but they are largely cancelled by the Fock terms. In the language of Ref. [16], the Fock terms contribute at NLO in $1 / N_{c}$ to an increased "stiffness" against mesonic fluctuations. This raises a question about the estimate of the critical stiffness in that work, which relies on an expression for the stiffness obtained from the LO pion propagator. This is in addition to the questions raised by other authors about the universality of the critical stiffness in 3+1 dimensions [37] and the need to choose an additional cut-off to regulate meson loops in the local NJL model [12,14].

The sigma meson is of particular interest since it can be excited by forces which act to restore manifest chiral symmetry, and its mass can be thought of as describing the forces against symmetry restoration. A light sigma seems to be a common feature in all NJL-type models at LO. We find that such a state is still present when NLO effects are included, although its mass is increased by about $30 \%$ (as one might expect from $1 / N_{c}$ arguments). For our preferred parameter sets we obtain a sigma mass in range $600-650 \mathrm{MeV}$. This suggests that the light sigma meson is a real feature of this type of model and not just an artefact of LO treatments.

However, the phenomenological identification of a corresponding low-lying scalar, isoscalar resonance remains controversial [31 33]. As discussed above, the problem is the strong coupling of such a state to the two-pion channel, leading to a very broad resonance. In our model we find a width of $100-160 \mathrm{MeV}$ from the LO coupling to two pions. While large, this width is much smaller than most phenomenological determinations [31 33]. We have not yet calculated NLO contributions to the $\sigma \pi \pi$ coupling, for which terms involving the vector mesons may prove to be important. In the extended model at NLO there will be $t$-channel exchange of a $\rho$ meson between the pions. The attractive force between the pions generated by this mechanism is known to be important for the sigma resonance [32,33 and could increase its decay width.

Finally, we have made a preliminary study of NLO effects in the extended model with vector and axial couplings. We find that NLO contributions, in particular from the longitudinal vector and axial channels, can improve the highenergy behaviour of the quark propagator. In general NLO effects seem to be significantly larger than in the simple version of the model, and the convergence of the $1 / N_{c}$ expansion may be less good as a result.

\section{ACKNOWLEDGEMENTS}

This work was supported by the EPSRC and PPARC.

\section{APPENDIX A: PION DECAY CONSTANT AT NLO}

Although the NLO contributions to the pion decay constant arise from a rather large set of diagrams, it is possible to deduce some useful cancellations amongst them. A complete description of these cancellations would be somewhat lengthy and so we offer only a few brief comments here. Full details can be found in Ref. [26].

Obviously the diagrams that couple the local axial current to the pion all contain an insertion of $\gamma_{\mu} \gamma_{5}$. Contracting this with $q^{\mu}$ to isolate the longitudinal component one can then substitute for $q \gamma_{5}$ from the following identity:

$$
\not d \gamma_{5}=S^{-1}\left(p_{+}\right) \gamma_{5}+\gamma_{5} S^{-1}\left(p_{-}\right)+\left(m\left(p_{+}\right)+m\left(p_{-}\right)\right) \gamma_{5} .
$$

The contributions from the first two terms here can be simplified by cancelling the LO inverse propagators with quark propagators that appear in the loop integrals.

Moreover, for each local diagram there is a similar nonlocal one which contains an additional one-quark loop. (For example, compare Fig. aa with $1 \mathrm{c}$ and Fig. $4 \mathrm{~b}$ with $4 \mathrm{~d}$.) These can be combined with the local contributions, as was done at LO [18]. Each of the NLO nonlocal diagrams can be written as a sum of products of two-quark and one-quark loops. In one of these terms, the one-quark loop can be simplified by using the ladder SDE to yield a factor proportional to $m(0)-m_{c}$. There is then a cancellation between this nonlocal term and the part of the corresponding local diagram arising from the final term of Eq. (A1) which leaves only $2 m_{c}$ surviving from the factor $m\left(p_{+}\right)+m\left(p_{-}\right)$ in Eq. (A1).

After exploiting Eq. A1) as outlined above, several other useful cancellations can be identified. As an example of

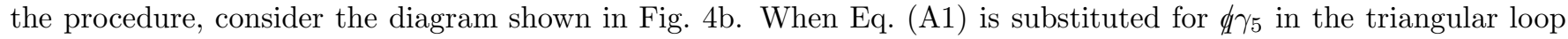
integral, the first two terms reduce to a sum of two-quark loops. Up to a factor of $-G J$ the resulting contributions are then similar in form to those of the diagram shown in Fig. 6a. 
Hence, in the sum of the contributions from Fig. $4 \mathrm{~b}$ and $6 \mathrm{a}$, one can cancel one of the intermediate meson propagators to leave a product of a two-quark loop, a three-quark loop and one meson propagator. It turns out that this can then be profitably combined with the contribution of Fig. $6 \mathrm{~b}$, which has the same structure.

We give here the result of a full analysis of the NLO contributions to $f_{\pi}$ in the version of the model with only scalar and pseudoscalar interactions. Note that, rather than defining further loop integrals whose structure is very similar to those given above in Sec. III d, we simply indicate how the products of form factors need to be changed in those integrals. The final expression for $f_{\pi}$ at NLO is 26]

$$
\begin{aligned}
& f_{\pi}^{(1)}\left(q^{2}\right)= \frac{i g_{\pi q q}^{(0)} G_{1}}{2 q^{2}} J_{\pi \pi}^{(1)}\left(q^{2}\right) \int \frac{d^{4} k}{(2 \pi)^{4}} \operatorname{Tr}[S(k)] f(k)(f(k+q)+f(k-q)) \\
&+\frac{g_{\pi q q}^{(0)} m_{c}}{q^{2}}\left(J_{\pi \pi}^{(1)} \text { from Eq. (20) with } f^{2}\left(p_{+}\right) f^{2}\left(p_{-}\right) \rightarrow f\left(p_{+}\right) f\left(p_{-}\right)\right) \\
&+\frac{g_{\pi q q}^{(0)} m_{c}}{q^{2}}\left(J_{\pi \pi}^{(1)} \text { from Eq. (21) with } f^{2}\left(p_{+}\right) f^{2}\left(p_{-}\right) \rightarrow f\left(p_{+}\right) f\left(p_{-}\right)\right) \\
&+\frac{g_{\pi q q}^{(0)} m_{c}}{q^{2}}\left(J_{\pi \pi}^{(1)} \text { from Eq. (24) with } f^{2}\left(p_{+}\right) f^{2}\left(p_{-}\right) \rightarrow f\left(p_{+}\right) f\left(p_{-}\right)\right. \\
&\left.\quad \text { in } \bar{L}_{\pi, k m}\right) \\
&-i \frac{C g_{\pi q q}^{(0)}}{2 q^{2}}\left(1-G_{1} J_{\pi \pi}^{(0)}\left(q^{2}\right)\right) \int \frac{d^{4} p}{(2 \pi)^{4}} \operatorname{Tr}[S(p) S(p)] f^{3}(p) \\
& \times \frac{g_{\pi q q}^{(0)}}{2 q^{2}}\left(1-G_{1} J_{\pi \pi}^{(0)}\left(q^{2}\right)\right) \sum_{i, j} \int \frac{d^{4} p}{(2 \pi)^{4}} \frac{d^{4} k}{(2 \pi)^{4}} \hat{T}_{i j}(p-k) \\
& \times \operatorname{Tr}\left[S(p) \Gamma_{i} S(k) \bar{\Gamma}_{j} S(p)\right] \\
& \times f^{3}(p)(f(p+q)+f(p-q)) f^{2}(k)
\end{aligned}
$$

\section{APPENDIX B: CHIRAL RELATIONS}

A large number of diagrams are required in a NLO treatment of the nonlocal NJL model, as described in Sec. III. It is important to have some check that a consistent set has been identified and evaluated. To this end, we demonstrate that low-energy chiral constraints are satisfied in the model at NLO. (Again we consider only the simpler version of the model, without vector and axial interactions.)

First consider the pion mass. The chiral expansion of the pion denominator, Eq. (25), at LO is [18]

$$
1-G_{1} J_{\pi \pi}^{(0)}\left(q^{2}\right)=-G_{1} m_{c} \frac{\langle\bar{\psi} \psi\rangle_{0}^{(0)}}{m_{0}(0)^{2}}-G_{1} \frac{q^{2}}{g_{\pi q q 0}^{(0) 2}}+\mathcal{O}\left(q^{4}, m_{c}^{2}\right),
$$

where the subscript 0 is used to denote a quantity evaluated in the chiral limit. (We apologize for the fact that the notation inevitably becomes rather cumbersome at this point.) If one substitutes the LO chiral expansion into the NLO on-shell condition, it is immediately clear that the chiral expansion of $J_{\pi \pi}^{(1)}\left(q^{2}\right)$ must start at $\mathcal{O}\left(q^{2}, m_{c}\right)$ in order for the pion to remain a Goldstone boson in the chiral limit.

A proof of this statement in the local NJL model was presented in Ref. [8]. Although one has to keep track of interaction form factors, the proof for the nonlocal model proceeds along very similar lines 26. In particular, when one takes the chiral limit of the contributions to $J_{\pi \pi}^{(1)}$ from Eqs. (20) and (24), each of the Dirac traces yields a factor that cancels with the denominator of one of the LO quark propagators. The three-quark loop in the contribution of the tadpole term to Eq. (20) can be reduced to a two-quark integral which can then be used to cancel the denominator 
of the $\sigma(0)$ propagator from Eq. (19). In the case of the contribution to $J_{\pi \pi}^{(1)}$ from Eq. (21), the Dirac trace takes an even more convenient form in the chiral limit. For both the sigma and pion exchanges, it factorizes into pieces which cancel the denominators from two LO quark propagators.

An important property noted in the proof of Ref. [8] concerns the second diagram of Fig. 3 which involves a virtual pion and a virtual sigma meson. In the chiral limit the three-quark loop integrals are proportional to the difference between LO polarization loops: $J_{\sigma \sigma}^{(0)}-J_{\pi \pi}^{(0)}$. This allows one to replace the product of the virtual meson propagators with their difference. It is then possible to combine the contribution from this diagram with other contributions, which contain only a single meson propagator. The same property holds in the nonlocal model

Many of the simplifications that lead to the vanishing of $J_{\pi \pi}^{(1)}$ in the chiral limit can also be exploited in calculating its $\mathcal{O}\left(m_{c}\right)$ and $\mathcal{O}\left(q^{2}\right)$ terms. One finds that 26]

$$
J_{\pi \pi}^{(1)}=-m_{c} \frac{\langle\bar{\psi} \psi\rangle_{0}^{(1)}}{m_{0}^{2}(0)}-m_{c} \frac{\langle\bar{\psi} \psi\rangle_{0}^{(0)}}{m_{0}^{2}(0)} \frac{2 C_{0}}{m_{0}(0)}-q^{2} \frac{2 g_{\pi q q 0}^{(1)}}{g_{\pi q q 0}^{(0) 3}}+\mathcal{O}\left(q^{4}, m_{c}^{2}\right)
$$

where the NLO contribution to the condensate is given by:

$$
\langle\bar{\psi} \psi\rangle^{(1)}=-i \operatorname{Tr} \int \frac{d^{4} p}{(2 \pi)^{4}} S(p) \Sigma^{(1)}(p) S(p) .
$$

Substituting the above expansion into the on-shell condition (Eq. (25)) yields the pion mass to this order,

$$
m_{\pi}^{2}=-\left(g_{\pi q q 0}^{(0)}+g_{\pi q q 0}^{(1)}\right)^{2} \frac{m_{c}\left[\langle\bar{\psi} \psi\rangle_{0}^{(0)}+\langle\bar{\psi} \psi\rangle_{0}^{(1)}\right]}{\left(m_{0}(0)+C_{0}\right)^{2}}+\mathcal{O}\left(m_{c}^{2}\right)+\mathcal{O}\left(N_{c}^{-2}\right)
$$

We see that the chiral expansion of the pion Bethe-Salpeter amplitude at NLO retains a similar structure to that at LO. The changes include the expected ones from the NLO contributions to the quark condensate and the pion-quark coupling. However, the form of the dynamical mass scale that appears in Eq. (B4) is less obvious, the shift in this being given entirely by the contribution of the tadpole diagram, Eq. (19). This is despite the fact that the meson cloud of a dressed quark contributes a term of $\mathcal{O}\left(m_{c}\right)$ to the chiral expansion of $J_{\pi \pi}^{(1)}$ and also makes a finite contribution to the quark self-energy at zero momentum. The fact that the NLO shift in the pion mass is controlled by the coefficient of the tadpole diagram has also been noted in the local NJL model [6.113, in which the overall mass scale can be recognized as the stationary point of the one-meson-loop effective action [6, 17 .

From Eq. (B4) it can be seen that the Gell-Mann-Oakes-Renner relation [25] will be satisfied if a version of the Goldberger-Treiman relation 38 holds in the chiral limit at NLO,

$$
\begin{aligned}
f_{\pi 0}^{(0)}+f_{\pi 0}^{(1)} & =\frac{m_{0}(0)+C_{0}}{g_{\pi q q 0}^{(0)}+g_{\pi q q 0}^{(1)}} \\
& =\frac{m_{0}(0)}{g_{\pi q q 0}^{(0)}}-\frac{g_{\pi q q 0}^{(1)}}{g_{\pi q q 0}^{(0)}} \frac{m_{0}(0)}{g_{\pi q q 0}^{(0)}}+\frac{C_{0}}{g_{\pi q q 0}^{(0)}}+\mathcal{O}\left(N_{c}^{-2}\right) .
\end{aligned}
$$

The term of order $N_{c}^{0}$ on the right-hand side of this condition was shown in Ref. [18] to be equal to the LO part of $f_{\pi}$.

In order to demonstrate that the NLO part of the decay constant reduces to the next two terms of Eq. (B5) in the chiral limit, first note that all of the contributions in Eq. A2 are of zeroth order in the chiral expansion. Hence in all of the integrals the chiral limit may be taken directly, without making an expansion of the integrand. In the first term of Eq. (A2) the ladder SDE can be used to perform the integral, giving

\footnotetext{
${ }^{6}$ It is in fact straightforward to generalize the proof that $J_{\pi \pi 0}^{(1)}(0)=0$ to the extended version of the model, including other mesons. In so doing the products of meson propagators occurring in diagrams of the type shown on the right-hand side of Fig. 3 can be dealt with using the relations $J_{\sigma \sigma}^{(0)}\left(q^{2}\right)-J_{\pi \pi}^{(0)}\left(q^{2}\right)=J_{V V}^{(0) T}\left(q^{2}\right)-J_{A A}^{(0) T}\left(q^{2}\right)=J_{V V}^{(0) L}\left(q^{2}\right)-J_{A A}^{(0) L}\left(q^{2}\right)$, where $T$ and $L$ denote transverse and longitudinal components respectively. Note, however, that in the extended model, one must take $\pi a_{1}$ mixing into account and so to complete the proof that the pion is a Goldstone boson at NLO, it is also necessary to establish that $J_{A \pi 0}^{(1)}(0)=0$.
} 


$$
-2 m_{0}(0) \frac{g_{\pi q q 0}^{(1)}}{g_{\pi q q 0}^{(0) 2}}+m_{c} \frac{g_{\pi q q 0}^{(0)}}{q^{2}} \frac{\langle\bar{\psi} \psi\rangle_{0}^{(1)}}{m_{0}(0)}-m_{c} \frac{g_{\pi q q 0}^{(0)}}{q^{2}} \frac{\langle\bar{\psi} \psi\rangle_{0}^{(0)}}{m_{0}(0)} \frac{2 C_{0}}{m_{0}(0)}
$$

where Eq. (B2) has been used for the chiral expansion of $J_{\pi \pi}^{(1)}$.

The next three terms in Eq. (A2) are explicitly proportional to $m_{c}$ and contain integrals which are very similar to those occurring in $J_{\pi \pi}^{(1)}$, but with different combinations of form factors. Procedures for manipulating such integrals in order to simplify their sum were described in Ref. [8] and also above, where they were exploited in order to show that $J_{\pi \pi}^{(1)}$ vanishes in the chiral limit. Very similar simplifications can be made here, despite the different form factor structures that appear. These give rise to a nonvanishing contribution to $f_{\pi 0}^{(1)}$,

$$
\frac{g_{\pi q q 0}^{(0)} m_{c}}{q^{2} m_{0}(0)}\left(-\langle\bar{\psi} \psi\rangle_{0}^{(1)}+\frac{C_{0}}{m_{0}(0)}\langle\bar{\psi} \psi\rangle_{0}^{(0)}\right)
$$

The fifth term in Eq. (A2) is proportional to the tadpole contribution $C$. Using Eq. (B1) to expand the factor of $1-G_{1} J_{\pi \pi}^{(0)}\left(q^{2}\right)$, the chiral limit of this term is

$$
\frac{g_{\pi q q 0}^{(0)}}{q^{2}} C_{0} G_{1} J_{\sigma \sigma 0}^{(0)}(0)\left(\frac{m_{c}\langle\bar{\psi} \psi\rangle_{0}^{(0)}}{m_{0}^{2}(0)}+\frac{q^{2}}{g_{\pi q q 0}^{(0) 2}}\right) .
$$

In the chiral limit, the integral in the sixth term of Eq. (A2) reduces to the one in the definition of $C$, Eq. (19), giving

$$
\frac{g_{\pi q q 0}^{(0)}}{q^{2}} C_{0}\left(1-G_{1} J_{\sigma \sigma 0}^{(0)}(0)\right)\left(\frac{m_{c}\langle\bar{\psi} \psi\rangle_{0}^{(0)}}{m_{0}^{2}(0)}+\frac{q^{2}}{g_{\pi q q 0}^{(0) 2}}\right) .
$$

The final term of Eq. ( $\mathrm{A} 2)$ is trivially

$$
\frac{g_{\pi q q 0}^{(1)}}{g_{\pi q q 0}^{(0)}} \frac{m_{0}(0)}{g_{\pi q q 0}^{(0)}}
$$

Adding together Eqs. ( $\mathrm{B} 6)$ to $(\mathrm{B} 10)$ reproduces exactly the $\mathcal{O}\left(N_{c}^{-1}\right)$ terms of Eq. (B5), demonstrating that the Gell-Mann-Oakes-Renner relation holds in our treatment of the nonlocal NJL model at NLO.

[1] Y. Nambu and G. Jona-Lasinio, Phys. Rev. 122 (1961) 345; 124 (1961) 246.

[2] U. Vogl and W. Weise, Prog. Part. Nucl. Phys. 27 (1991) 195; S. P. Klevansky, Rev. Mod. Phys. 64 (1992) 649; T. Hatsuda and T. Kunihiro, Phys. Rep. 247 (1994) 221; J. Bijnens, Phys. Rep. 265 (1996) 369.

[3] E. Quack and S. P. Klevansky, Phys. Rev. C49 (1994) 3283.

[4] A. Polleri, R. A. Broglia, P. M. Pizzochero and N. N. Scoccola, Z. Phys. A357 (1997) 325.

[5] N.-W. Cao, C. M. Shakin and W.-D. Sun, Phys. Rev. C46 (1992) 2535; P. P. Domitrovich, D. Bückers and H. Müther, Phys. Rev. C48 (1993) 413; P. Zhuang, J. Hüfner and S. P. Klevansky, Nucl. Phys. A576 (1994) 525; P. Zhuang, J. Hüfner, S. P. Klevansky and H. Voss, Ann. Phys. (N.Y.) 234 (1994) 225; Y. B. He, J. Hüfner, S. P. Klevansky and P. Rehberg, Nucl. Phys. A630 (1998) 719.

[6] E. Nikolov, W. Broniowski, C. V. Christov, G. Ripka and K. Goeke, Nucl. Phys. A608 (1996) 411.

[7] R. D. Bowler, Ph.D. thesis, University of Manchester, 1995.

[8] V. Dmitrašinović, H.-J. Schulze, R. Tegen and R. H. Lemmer, Ann. Phys. (N.Y.) 238 (1995) 332.

[9] E. Pallante, Z. Phys. C75 (1997) 305.

[10] M. Huang, P. Zhuang and W. Chao, Commun. Theor. Phys. 34 (2000) 91.

[11] B. Szczerbinska and W. Broniowski, Acta Phys. Polon. B31 (2000) 835; D. Gómez Dumm and N. N. Scoccola, hepph/0107251 (2001).

[12] M. Oertel, M. Buballa and J. Wambach, Phys. Lett. B477 (2000) 77.

[13] M. Oertel, M. Buballa and J. Wambach, Nucl. Phys. A676 (2000) 247. 
[14] G. Ripka, Nucl. Phys. A683 (2001) 463; hep-ph/0007250 (2000).

[15] M. Oertel, Ph.D. thesis, University of Darmstadt, 2000.

[16] H. Kleinert and B. Van den Bossche, Phys. Lett. B474 (2000) 336; hep-ph/9908284 (1999).

[17] M. Oertel, M. Buballa and J. Wambach, Phys. Atom. Nucl. 64 (2001) 698; Yad. Fiz. 64 (2001) 757.

[18] R. D. Bowler and M. C. Birse, Nucl. Phys. A582 (1995) 655.

[19] R. S. Plant and M. C. Birse, Nucl. Phys. A628 (1998) 607.

[20] W. Broniowski, AIP Conf. Proc. 508 (2000) 380.

[21] B. Golli, W. Broniowski and G. Ripka, Phys. Lett. B437 (1998) 24; W. Broniowski, hep-ph/9909438 (1999); G. Ripka and B. Golli, AIP Conf. Proc. 508 (2000) 3; W. Broniowski, B. Golli and G. Ripka, hep-ph/0107139 (2001).

[22] D. I. Dyakonov and V. Yu. Petrov, Nucl. Phys. B245 (1984) 259; Sov. Phys. JETP 62 (1985) 204, 431; Nucl. Phys. B272 (1986) 457.

[23] M. Musakhanov, Eur. Phys. J. C9 (1999) 235.

[24] K. Naito, K. Yoshida, Y. Nemoto, M. Oka and M. Takizawa, Phys. Rev. C59 (1999) 1095.

[25] M. Gell-Mann, R. Oakes and B. Renner, Phys. Rev. 175 (1968) 2195.

[26] R. S. Plant, Ph.D. thesis, University of Manchester, 1998.

[27] D. Blaschke, Yu. L. Kalinovsky, G. Röpke, S. Schmidt and M. K. Volkov, Phys. Rev. C53 (1996) 2394.

[28] C. Itzykson and J.-B. Zuber, Quantum Field Theory (McGraw-Hill 1980).

[29] K. L. Au, D. Morgan and M. Pennington, Phys. Rev. D35 (1987) 1633; D. Morgan and M. R. Pennington, Phys. Rev. D48 (1993) 1185.

[30] B. S. Zou and D. V. Bugg, Phys. Rev. D50 (1994) 591; R. Kaminski, L. Lesniak and J.-P. Maillet, Phys. Rev. D50 (1994) 3145; S. Ishida et al., Prog. Theor. Phys. 95 (1996) 745; ibid. 98 (1997) 1005; N. A. Tornqvist and M. Roos, Phys. Rev. Lett. 76 (1996) 1575; M. Harada, F. Sannino and J. Schechter, Phys. Rev. D54 (1996) 1991; R. Kaminski, L. Lesniak and B. Loiseau, Phys. Lett. B413 (1997) 130; Eur. Phys. J. C9 (1999) 141; T. Hannah, Phys. Rev. D60 (1999) 017502; M. Y. Ishida et al., hep-ph/9905261 (1999); V.V. Anisovich and V. A. Nikonov, hep-ph/9911512 (1999).

[31] Particle Data Group, Eur. Phys. J. C3 (1998) 1.

[32] M. R. Pennington, hep-ph/9905241 (1999).

[33] V. E. Markushin and M. P. Locher, hep-ph/9906249 (1999).

[34] M. Oertel (private communication).

[35] G. V. Efimov and M. A. Ivanov, The Quark Confinement Model of Hadrons (IOP, Bristol and Philadelphia, 1993).

[36] L. S. Celenza, B. Huang, H. Weng and C. M. Shakin, Phys. Rev. C60 (1999) 065210; L. S. Celenza, S.-F. Gao, B. Huang, H. Weng and C. M. Shakin, Phys. Rev. C61 (2000) 035201.

[37] E. Babaev, Phys. Rev. D62 (2000) 074020.

[38] M. Goldberger and S. Treiman, Phys. Rev. 110 (1958) 1178, 1478. 


\section{APPENDIX: TABLES}

\begin{tabular}{cccccc}
\hline \hline$m_{0}(0)$ & $G_{1}\left(\mathrm{GeV}^{-2}\right)$ & $m_{c}$ & $\Lambda$ & $m(0)$ & $-\langle\bar{q} q\rangle^{1 / 3}$ \\
\hline 200 & 14.3 & 4.8 & 1459 & 245 & 246 \\
250 & 30.5 & 7.8 & 1064 & 298 & 210 \\
300 & 53.8 & 11.0 & 861 & 351 & 189 \\
350 & 85.9 & 14.2 & 734 & 406 & 3.13 \\
400 & 128.1 & 17.5 & 647 & 461 & 173 \\
450 & 181.7 & 20.8 & 583 & 516 & 162 \\
500 & 248.0 & 24.1 & 535 & 572 & 153 \\
\hline \hline
\end{tabular}

TABLE I. Values of the model parameters, fitted at LO. Also listed are the dynamical quark mass, the quark condensate in the chiral limit and the pion-quark coupling. Apart from $G_{1}$ and $g_{\pi q q}$, which is dimensionless, all quantities are given in MeV.

\begin{tabular}{cccrrr}
\hline \hline$m_{0}(0)$ & $-\langle\bar{q} q\rangle^{1 / 3}$ & $C$ & $C_{0}$ & $C_{0}(\pi)$ & $C_{0}(\sigma)$ \\
\hline 200 & 259 & 25.0 & 25.3 & 90.2 & -64.9 \\
250 & 215 & 12.4 & 8.0 & 22.4 & -36.2 \\
300 & 190 & 5.2 & -1.7 & 8.6 & -24.1 \\
350 & 174 & 0.005 & -8.8 & -1.4 & -17.4 \\
400 & 162 & -4.1 & -14.6 & -9.5 & -13.1 \\
450 & 153 & -7.7 & -19.6 & -16.3 & -7.9 \\
500 & 145 & -10.8 & -24.2 & -2 \\
\hline \hline
\end{tabular}

TABLE II. Properties of the quark propagator at NLO. All quantities are quoted in MeV. The quark condensate is evaluated in the chiral limit. The constant $C$ appears in the part of the self-energy from the NLO tadpole diagram and is defined in Eq. (19). Its value in the chiral limit is $C_{0}$. The contribution to $C$ from the tadpole diagram with a virtual meson $m$ is denoted $C(m)$.

\begin{tabular}{ccccc}
\hline \hline$m_{0}(0)$ & $m_{\sigma}^{(0)}$ & $g_{\sigma \pi \pi}^{(0)}$ & $\Gamma^{(0)}(\sigma \rightarrow \pi \pi)$ & $\mathrm{Re}\left[m_{\sigma}\right]$ \\
\hline 200 & 385 & 1092 & 63.5 & 455 \\
250 & 423 & 1336 & 94.4 & 552 \\
300 & 454 & 1562 & 126.3 & 624 \\
350 & 477 & 1732 & 152.0 & 650 \\
400 & 492 & 1783 & 158.7 & $>574$ \\
450 & 489 & 1489 & 111.0 & $>504$ \\
500 & 478 & 1515 & 116.2 & $>450$ \\
\hline \hline
\end{tabular}

TABLE III. Sigma meson mass evaluated at NLO. For comparison the mass and other properties at LO are also given (denoted by a superscript (0)). All quantities are quoted in $\mathrm{MeV}$. For the parameter sets with large $m_{0}(0)$, the energy at which pseudo-threshold effects are required 19] is rather modest, and hence we quote only a lower bound for the mass at NLO. 


\section{APPENDIX: FIGURES}
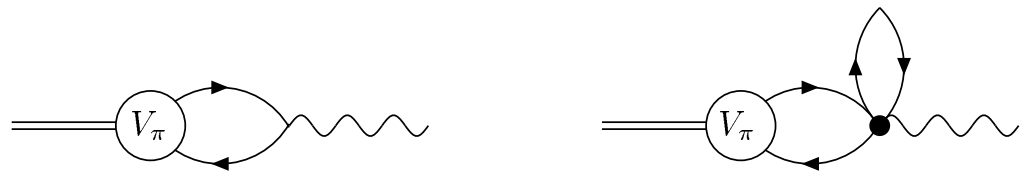

FIG. 1. The LO couplings of a pion to the axial current (represented by the wavy line).
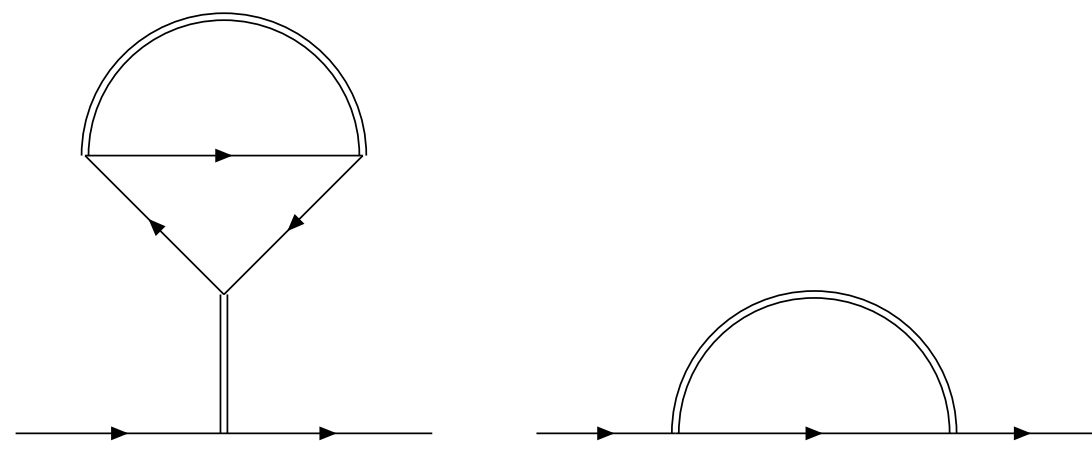

FIG. 2. Diagrams contributing to the NLO quark self-energy. Double lines are used to denote the LO $\bar{q} q$ scattering matrix.
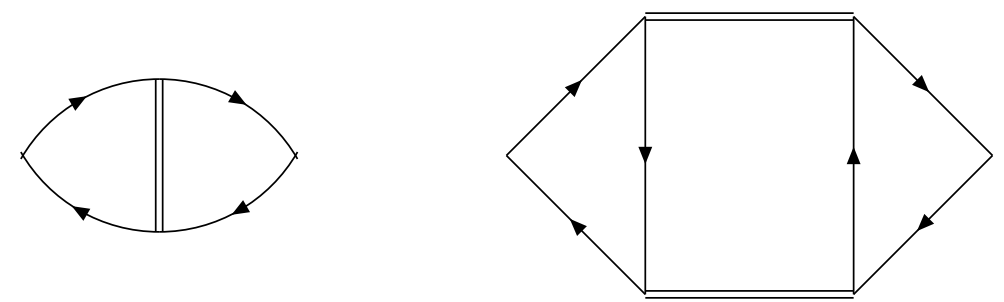

FIG. 3. Meson exchange and two-meson diagrams in the BSE at NLO. There is also a second two-meson diagram (not shown) in which the direction of the arrows in one of the quark loops is reversed. 


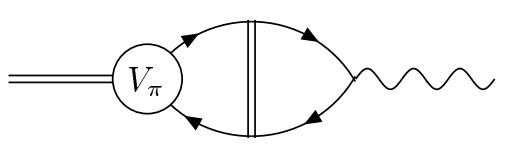

(a)

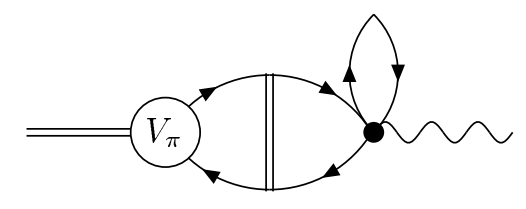

(c)

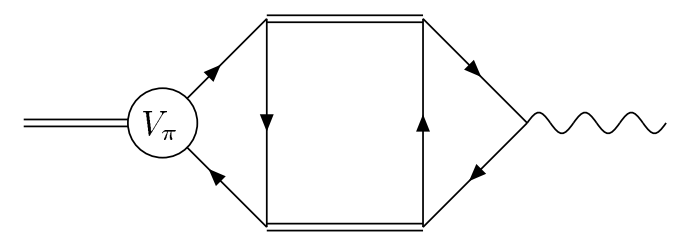

(b)

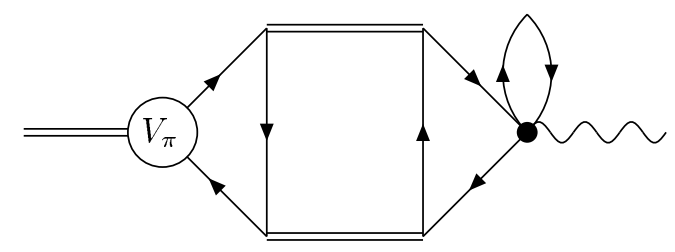

(d)

FIG. 4. NLO couplings of a pion to the axial current involving either meson exchange or two-meson intermediate states. There are also two-meson diagrams in which the direction of the arrows in one of the quark loops is reversed.

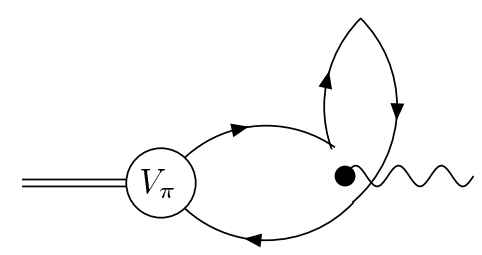

FIG. 5. The exchange contribution to the coupling of the pion to the nonlocal axial current. Note that this Fock diagram has been distinguished from the similar LO diagram by separating the quark lines to indicate the flow of colour.

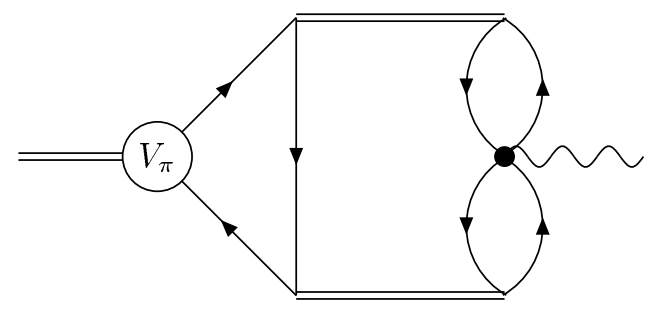

(a)

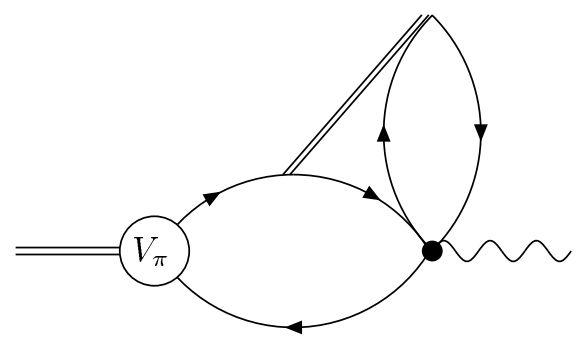

(b)

FIG. 6. NLO contributions to the coupling of a pion to the nonlocal axial current involving a virtual meson. There is also a second diagram similar to (b) in which the virtual meson couples to the antiquark line. 


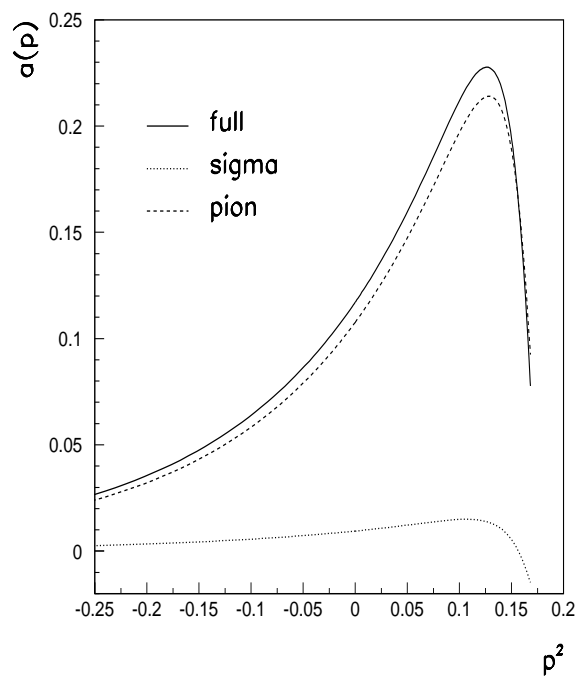

FIG. 7. The function $a(p)$ in the quark propagator at NLO, plotted against $p^{2}$ in $\mathrm{GeV}^{2}$. Also shown are the contributions to $a(p)$ obtained by dressing the quark with pion and sigma clouds separately. The parameter set used is the one with $m_{0}(0)=300$ $\mathrm{MeV}$ in Table If.

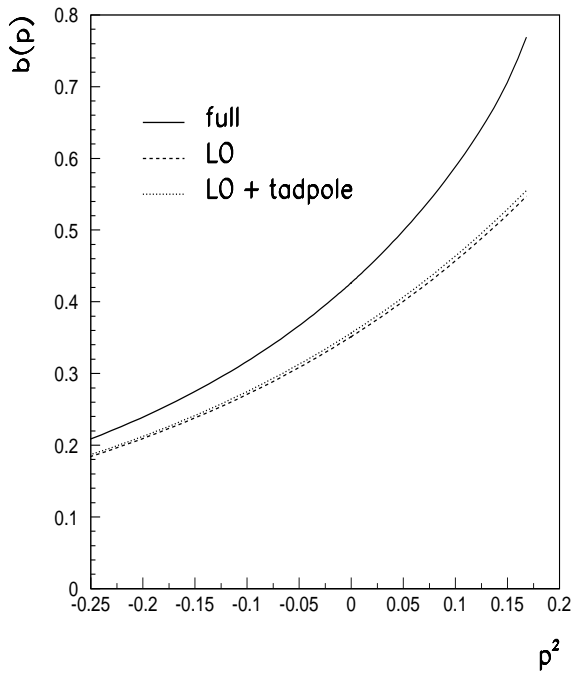

FIG. 8. The function $b(p)$ in the NLO quark propagator, plotted against $p^{2}$ in $\mathrm{GeV}^{2}$. Also shown are the LO contribution and the sum of the LO result and the tadpole contributions. The parameter set is used is the one with $m_{0}(0)=300 \mathrm{MeV}$ in Table I 


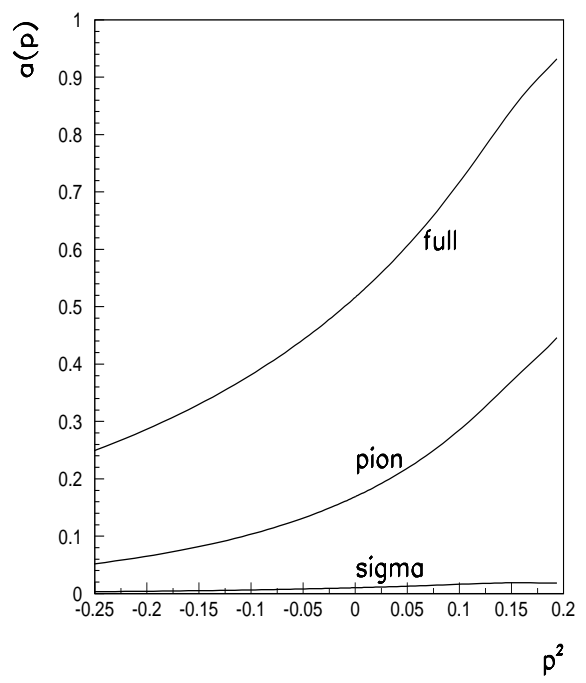

FIG. 9. The function $a(p)$ in the quark propagator of the extended model, plotted against $p^{2}$ in $\mathrm{GeV}^{2}$. The full NLO result is shown together with the contributions from the pion and sigma clouds.

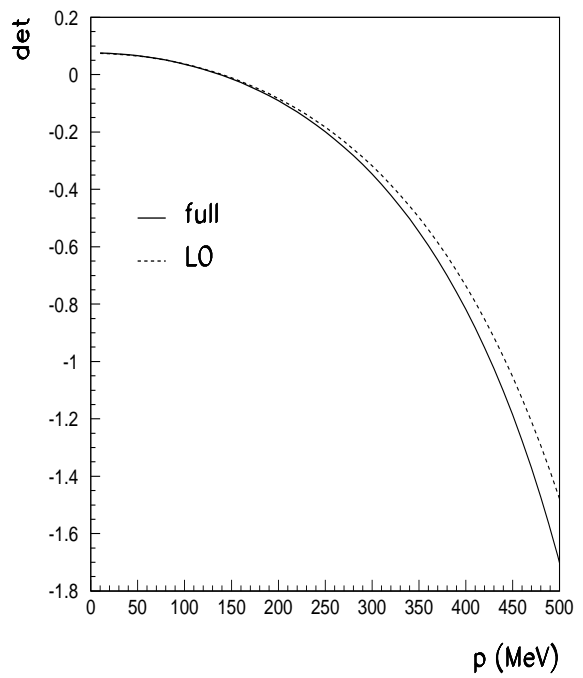

FIG. 10. The denominator of the scattering matrix in the pion channel at LO and NLO as a function of timelike momentum. The parameter set used is the one with $m_{0}(0)=300 \mathrm{MeV}$ in Table If. 


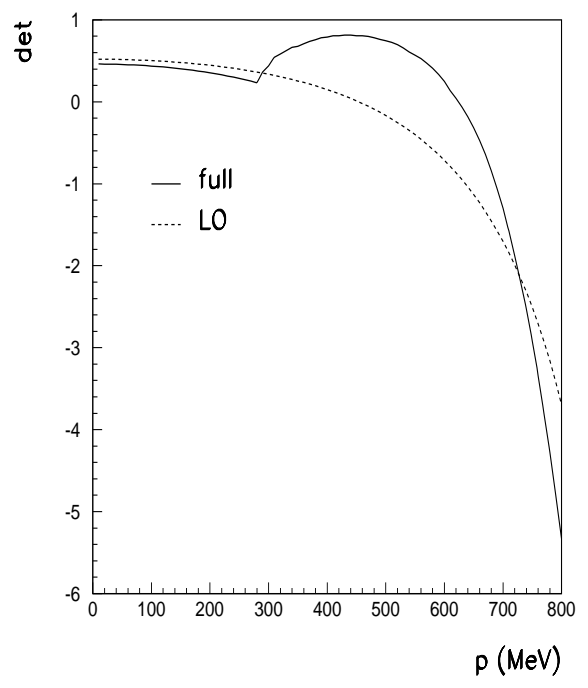

FIG. 11. The real part of the denominator of the scattering matrix in the sigma channel at LO and NLO as a function of timelike momentum. The parameter set used is the one with $m_{0}(0)=300 \mathrm{MeV}$ in Table $\mathrm{f}$.

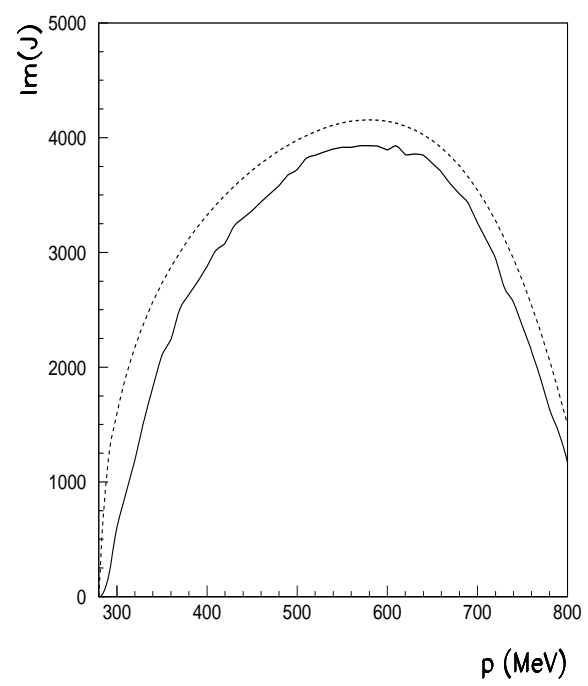

FIG. 12. The imaginary part (in $\mathrm{MeV}^{2}$ ) at NLO of the loop $J$ in the sigma channel as a function of timelike momentum.

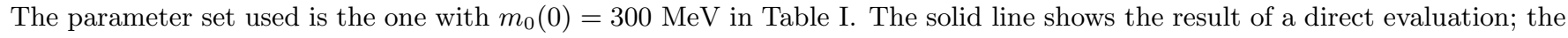
dashed line an estimate based on the Cutkosky cutting rules. 